\title{
A discrete-time terminal sliding mode controller design for an autonomous underwater vehicle
}

\author{
Nira Mawangi Sarif', Rafidah Ngadengon ${ }^{2}$, Herdawati Abdul Kadir ${ }^{3}$, Mohd Hafiz A. Jalil ${ }^{4}$, \\ Khalid Abidi ${ }^{5}$ \\ 1,2,3,4 Faculty of Electrical \& Electronic Engineering, University Tun Hussein Onn Malaysia, Malaysia \\ ${ }^{5}$ Newcastle University, Singapore
}

\begin{tabular}{l} 
Article Info \\
\hline Article history: \\
Received Sep 26, 2020 \\
Revised Dec 1, 2020 \\
Accepted Feb 20, 2021 \\
\hline
\end{tabular}

Keywords:

Autonomous underwater vehicle

Chattering reduction

Convergence acceleration

Digital control

Fractional power rule

Sliding mode control

Terminal sliding mode control

\begin{abstract}
Autonomous underwater vehicle (AUV) are underwater robotic devices intended to explore hostiles territories in underwater domain. AUVs research gaining popularity among underwater research community because of its extensive applications and challenges to overcome unpredictable ocean behavior. The aim of this paper is to design discrete time terminal sliding mode control (DTSMC) reaching law-based employed to NPS AUV II purposely to improve the dynamic response of the closed loop system. This is accomplished by introducing a nonlinear component to sliding surface design in which the system state accelerated, and chattering effect is suppressed. The nonlinear component consist of fractional power is to ensure steeper slope of the sliding surface in the vicinity of the equilibrium point which lead to quicker convergence speed. Thus, the chattering effect in the control action suppressed as the convergence of the system state accelerated. The stability of the control system is proven by using Sarpturk analysis and the performance of the DTSMC is demonstrated through simulation study. The performance of DTSMC is benchmarked with DSMC and PID controller.
\end{abstract}

This is an open access article under the $\underline{C C B Y-S A}$ license.

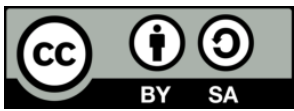

\section{Corresponding Author:}

Rafidah Ngadengon

Faculty of Electrical \& Electronic Engineering

Univeristi Tun Hussein Onn Malaysia

86400 Parit Raja, Batu Pahat, Johor, Malaysia

Email: rafida@uthm.edu.my

\section{INTRODUCTION}

Autonomous underwater vehicle (AUV) is used for autonomous mission under hundreds of thousands of meters depth ranges for tens of hour [1-8]. Owing good working capabilities in deep ocean, AUV has been successfully applied in military, underwater inspection of oil/gas pipelines and risers, ocean forecasting and ecosystem monitoring. A typical AUV problem is position tracking control which aim to steer the AUV to follow desired position precisely [2, 9]. However, due to AUV complex dynamics, nonlinearity, parametric uncertainties, external disturbances, and measurement noise the controller performance degraded. To solve the control problem of AUV, many kind of controllers, namely linear [3-5] intelligent $[6,7]$ and robust controller $[10,11]$ method have been executed for the AUV position tracking control problem. Although the linear controller such as proportional-integral-derivative (PID) could established with acceptable performance, real AUV applications face a lot of uncertainties in the complex ocean environments. The PID alone however does not robust to those uncertainties [3-5]. Nevertheless, various kind of auto gain tuning method were introduced in $[12,13]$ to improve PID effectiveness on AUV. However, the performance is sensitive to specific requirement, model accuracy and disturbance characteristic. For this reason, several study on intelligent control method such as fuzzy logic (FL) [12-14] 
and neural network (NN) [15-17] dedicated to AUV shows suitable performance. FL and NN, required expert experience about the fuzzy rules and sample training for $\mathrm{NN}[18,19]$. Thus, it's difficult to implement in real time for AUV control system [20,21].

Sliding mode control (SMC) has shown robustness and efficient way to cope with system disturbances in comparison to other control approaches [20]. The essence of sliding mode control is to force the system state to converge to sliding surface and remain on it for subsequent time. Once the sliding surface intercepted, the behaviour of the dynamic system governed by the sliding mode phase. Thus, the dynamic system become stable and robust to system variation. However, during sliding mode phase, undesirable high frequency oscillation in switching frequency results chattering motions which deteriorates SMC performance. As reported in literature, chattering reduction of reaching law-based is obtained by choosing small control gain. However, small control gains lead to longer reaching phase and poor robustness [21]. Moreover, the system state convergence to equilibrium point is asymptotically due to linear property of the classical SMC. The asymptotical stability may not deliver fast convergence without strong control force. To overcome the shortcoming, various effort has been proposed to improve convergence process and reduce chattering effect at the same time. For instant, novel reaching law introduced in [22], higher order sliding surface [23], integral sliding surface [24], nonlinear sliding surface [25, 26] so on and so forth. In this research, nonlinear sliding surface is presented in order to improve the state convergence acceleration and reduce the chattering effect thus the dynamic response of the closed loop system can be improved in comparison to classical SMC and commercial PID controller.

Terminal sliding mode control (TSMC) introduced in early 1990s is one of nonlinear sliding surface. The term "terminal" referred to the equilibrium point as the terminal attractor in which the system state reached the equilibrium point in finite time and stable. By introducing the nonlinear power rule in the sliding surface design, the convergence rate of the system state accelerated once the sliding surface intercepted and the chattering effect is suppressed. As a result, the transient response of TSMC improves substantially. In recent work, DTSMC has been used successfully in AUV control design to achieve fast and finite time convergence [27-32] and mostly are in continuous time domain. However, due to technology advancement, the effectiveness of continuous SMC in real applications remains inadequate [33, 34]. For this reason, discrete time terminal sliding mode control (DTSMC) application become reasonable [35-39] to AUV control design.

\section{MODEL OF NPS AUV II}

\subsection{Nonlinear equation of motion}

Considering Newton-Euler formulation, the 6-DOF nonlinear equation of motion (EOM) of AUVs are defined as

$$
M \dot{v}+C(v) v+D(v) v+g(n)=\tau
$$

where, $M \in \mathfrak{R}^{6 \times 6}$ is the inertia matrix of AUVs rigid body including added mass, $C_{R B}(v) \in \mathfrak{R}^{6 \times 6}$ is the Coriolis and centripetal dynamics matrix including added mass, $D(v) \in \mathfrak{R}^{6 \times 6}$ is the damping matrix that consists of the drag and lift terms, $g(n) \in \mathfrak{R}^{6}$ is the restoring forces and moment vector which contain gravitational and buoyancy forces and $\tau \in \mathfrak{R}^{6}$ is the vector of control inputs relating to the forces and moments acting on the AUV. Fossen in [40] proposed a AUV formulation using a society of naval architects and marine engineers (SNME) standard where two coordinate system known as the body fixed frame (BFF) and the Earth Fixed Frame (EFF) are introduced for convenience as shown in Figure 1 and Table 1. Notation vectors in BFF are defined as

$$
\begin{aligned}
& v=\left[\begin{array}{ll}
v_{1} & v_{2}
\end{array}\right]^{T} \\
& v_{1}=\left[\begin{array}{lll}
u & v & w
\end{array}\right]^{T} \text { Linear velocities } \\
& v_{2}=\left[\begin{array}{lll}
p & q & r
\end{array}\right]^{T} \text { Angular velocities }
\end{aligned}
$$

The notation vector in EFF are defined as:

$$
\begin{aligned}
& n=\left[\begin{array}{ll}
n_{1} & n_{2}
\end{array}\right]^{T} \\
& n_{1}=\left[\begin{array}{lll}
x & y & z
\end{array}\right]^{T} \in \mathfrak{R}^{3} \text { Position Of Origin } \\
& n_{2}=\left[\begin{array}{lll}
\phi & \theta & \psi
\end{array}\right]^{T} \in \mathfrak{R}^{3} \text { Angles orientation of roll, pitch and yaw }
\end{aligned}
$$

A discrete-time terminal sliding mode controller design for an autonomous ...(Nira Mawangi Sarif) 
Force and torque control input vector is defined as

$$
\tau=\left[\begin{array}{llllll}
X & Y & Z & K & M & N
\end{array}\right]^{T}
$$

The 6-DOF nonlinear EoM and coupled equations lead to complexcities in the controller design. Therefore, the 6-DOF EoM is divided into three non-interacting subsystems [41, 42]. Grouping certain key motion equations together and considering the rolling mode $p(t)$ and $\phi(t)$ is passive, the decoupled subsystems are indicated in Table 2.

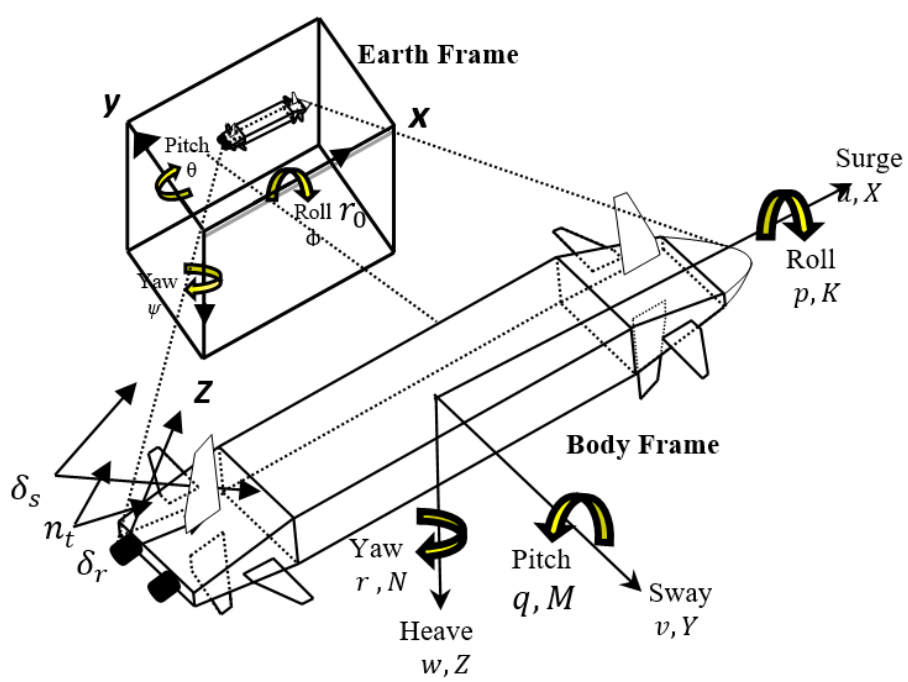

Figure 1. The schematic of NPS AUV II BFF and EFF [40]

Table 1. The notation of NPS AUV II for marine vessel [40]

\begin{tabular}{cccc}
\hline Degree of freedom & Forces \& moments & Linear and angular velocities & Position and euler angles \\
\hline Surge & $X$ & $u$ & $x$ \\
Sway & $Y$ & $v$ & $y$ \\
Heave & $Z$ & $w$ & $z$ \\
Roll & $K$ & $p$ & $\phi$ \\
Pitch & $M$ & $q$ & $\theta$ \\
Yaw & $N$ & $r$ & $\psi$ \\
\hline
\end{tabular}

Table 2. The notations of NPS AUV II for marine vessel [40]

\begin{tabular}{lcc}
\hline \multicolumn{1}{c}{ Subsystem } & Description & Control Input \\
\hline Speed & $u(t)$ & $n(t)$ \\
Steering & $v(t), r(t), \psi(t)$ & $\delta_{r}(t)$ \\
Diving & $w(t), q(t), \theta(t), z(t)$ & $\delta_{s}(t)$ \\
\hline
\end{tabular}

\subsection{Pitch and depth (motion in diving plane) linearized equation of motion}

This research focus on the diving autopilot controller design. For this reason, steering parameters are set to zero ( $v=p=r=\phi=\psi=0$ ) where diving EOM are focus on heave velocity $w$, the angular velocity in pitch $q$, the pitch angle $\theta$, the depth $z$ and the elevator plane deflection $\delta_{s}$ are considered. Noting that the NPS AUV II moves very slow during vertical direction [43-48]. Therefore, heave velocity $w$ is considered negligible. Simplified nonlinear dynamics EOM is then given as

$$
\begin{aligned}
& \dot{q}=\frac{M_{u q} u q-W \overline{B G_{z}} \sin \theta+M_{\delta} \delta_{s}}{I_{y}-M_{\dot{q}}} \\
& \dot{\theta}=q \cos \phi \\
& \dot{z}=-u \sin \theta
\end{aligned}
$$


During steady state motion, forward velocity is constant $\left(u=u_{o}\right)$, pitch angle is constant $(\sin \theta=$ $\left.\theta_{0}\right)$ and pitch velocity is zero $\left(q_{0}=0\right)$. Therefore, the linearized equation of motion for diving plane is written as:

$$
\begin{aligned}
& \dot{q}=\frac{M_{\dot{q}}-W \overline{B G_{z}} \theta+M_{\delta} \delta_{s}}{I_{y}-M_{\dot{q}}} \\
& \dot{\theta}=q \\
& \dot{z}=-\theta u_{0}
\end{aligned}
$$

The diving linearized model in (12), (13) and (14) can be transform to state space model by choosing the output as $y=z$.

$$
\begin{aligned}
& \dot{x}(t)=A x(t)+B \delta_{s}(t)+B d(t) \\
& y(t)=C x(t)
\end{aligned}
$$

with $x=\left[\begin{array}{lll}q & \theta & z\end{array}\right]^{T} \in \mathfrak{R}^{3}, \delta_{s} \in \mathfrak{R}$. Where $C=\left[\begin{array}{lll}0 & 0 & 1\end{array}\right] \in \mathfrak{R}^{3}$. The matrices $A$ and $B$ are the state and input matrices respectively, $d \in \mathfrak{R}$ is the lumped disturbance [46] as (16).

$$
d(t)=0.1 \sin 0.02 t
$$

Finally, the diving motion linear model is reduced to the form

$$
\left[\begin{array}{c}
\dot{q} \\
\dot{\theta} \\
\dot{z}
\end{array}\right]=\left[\begin{array}{ccc}
\frac{M_{q}}{I_{y}-M_{\dot{q}}} & \frac{-W\left(Z_{G}-Z_{B}\right)}{I_{y}-M_{\dot{q}}} & 0 \\
1 & 0 & 0 \\
0 & -u_{0} & 0
\end{array}\right]\left[\begin{array}{l}
q \\
\theta \\
z
\end{array}\right]+\left[\begin{array}{c}
\frac{M_{\delta}}{I_{y}-M_{\dot{q}}} \\
0 \\
0
\end{array}\right] \delta_{s}+\left[\begin{array}{c}
\frac{M_{\delta}}{I_{y}-M_{\dot{q}}} \\
0 \\
0
\end{array}\right] d
$$

where $M_{q}$ is the pitch moment due to pitch velocity, $M_{\dot{q}}$ is the pitch moment due to the rate of change of pitch velocity, $I_{y}$ is the vehicle inertia around the pitch axes, $W$ is the AUV weight, $Z_{G}$ is the center of gravity, $Z_{B}$ is the center of buoyancy, $u_{0}$ is the velocity and $M_{\delta}$ is the elevator lift coefficient.

\section{DISCRETE TIME SLIDING MODE CONTROL FOR NPS AUV II}

In this section, the discrete-time SMC approach is employed to design digital control for NPS AUV II during diving motion. First, the zero_order_hold ( $\mathrm{ZOH})$ is used to obtain the discrete-time model of system in (17) with a sampling interval of $T$ which gives

$$
\begin{aligned}
& x(k+1)=\Phi x(k)+\Gamma \delta_{s}(k)+\Gamma f(k) \\
& y(k)=C x(k)
\end{aligned}
$$

where $x(k) \in \mathfrak{R}^{3}$ is the state and $\delta_{s} \in \mathfrak{R}$ is the control input vector and $\Phi \in \mathfrak{R}^{3 \times 3}$ and $\Gamma \in \mathfrak{R}^{3}$ are the system matrices. The matrices of $\Phi$ and $\Gamma$ are in appropriate dimension and $f(k) \in \Re$ results from parametric uncertainties and exogenous disturbances. Above matrices and vectors are defined as

$$
\begin{aligned}
& \Phi=e^{A_{p} t}, \quad \Gamma=\int_{0}^{\tau} e^{A_{p} t} B_{p} d t \\
& f(k)=(C T)^{-1} C \int_{0}^{\tau} e^{A_{p} t} B_{p} f((k+1) \tau-\tau) d \tau
\end{aligned}
$$

where $A_{p}$ and $B_{p}$ are the nominal values of the matrices $A$ and $B$ respectively. The disturbance $f(k)$, in the discrete time system is represented as

$$
f(k)=\Delta \bar{\Phi} x(k)+\Delta \bar{\Gamma} \delta_{s}(k)+d(k)+O\left(T^{3}\right)
$$


where $\Delta \bar{\Phi} \in \mathfrak{R}^{3}$ and $\Delta \bar{\Gamma} \in \mathfrak{R}^{3}$ are vectors of parameter uncertainties and $O(\cdot)$ is the order of '.' [27]. Based on discrete-time model (18), both the DSMC law and the DTSMC law are designed to improve the transient response of the system and reducing chattering effect in the control input.

\subsection{Designing discrete time SMC law (DSMC)}

For discrete time system in (18), the sliding mode surface is linear and chosen as

$$
S(k)=C_{s} e(k)=C_{S}\left(x_{r}(k)-x(k)\right)
$$

where $e(k)$ is the position tracking error, $x_{r}$ is the reference input and $C_{s}$ is the sliding matrix which is to be designed for the best possible performance of the system.

Since it is desirable to have a robust controller, therefore DSMC law is obtained by combining equivalent control method with Gao's reaching law [47] as

$$
S(k+1)=(1-\alpha T) S(k)-\varepsilon \operatorname{Tsgn}(S(k))
$$

where $T$ is the sampling interval of discrete time system, $\varepsilon>0, \alpha>0$ and $|1-\alpha T|<1$. From (18), (20), (21) and (22) the discrete time sliding mode dynamics is defined as

$$
S(k+1)=\left[\begin{array}{c}
C_{s} \Phi x(k)+C_{s} \Gamma f(k)+C_{s} x_{r}(k+1)+C_{s} \delta_{s}(k) \\
-(1-\alpha T) S(k)+\varepsilon T \operatorname{sgn}(S(k))
\end{array}\right]
$$

The sliding mode is obtained when $S(k+1)=0$ is achieved. By applying this condition into (23) and considering matching condition are satisfied as in [47] the DSMC control law defined as

$$
\delta_{s}(k)=-\left(C_{s} \Gamma\right)^{-1}\left[\begin{array}{c}
-C_{s} x_{r}(k+1)+C_{s} \Phi x(k)+(1-\alpha T) S(k) \\
-\varepsilon T s g n(S(k))+f_{0}+\delta_{0} \operatorname{sgn}(S(k))
\end{array}\right]
$$

where $\boldsymbol{f}_{\mathbf{0}}$ is the average value of disturbance and $\boldsymbol{\delta}_{\mathbf{0}}$ is the maximum admissible deviation of disturbance in (20).

\subsection{Design discrete time terminal SMC law (DTSMC)}

In this section, DTSMC law is designed to improve the convergence acceleration of the position tracking error. Firstly, the nonlinear sliding surface is designed as:

$$
S_{T}(k)=C_{T s} e(k)+\beta e_{3}^{\frac{q_{c}}{p_{c}}}(k)
$$

where $\beta>0, p_{c}$ and $q_{c}$ are both positive odd integers such that $p_{c}>q_{c}, 0<\frac{q_{c}}{p_{c}}<1$ and $C_{T s}$ is the terminal sliding matrix which is to be designed for the best possible performance.

Replacing (18), (20), (22) and (25), the discrete time terminal sliding mode dynamics is defined as:

$$
S_{T}(k+1)=\left[\begin{array}{c}
C_{T s} \Phi x(k)+C_{T s} \Gamma f(k)+C_{T s} x_{r}(k+1)+C_{T s} \delta_{s}(k) \\
+\beta x_{3}{ }^{\frac{q_{c}}{p_{c}}}(k)-(1-\alpha T) S(k)+\varepsilon T s g n(S(k))
\end{array}\right]
$$

By applying similar method as in section, A, DTSMC law is obtained as:

$$
\delta_{s}(k)=-\left(C_{T s} \Gamma\right)^{-1}\left[\begin{array}{c}
-C_{T s} x_{r}(k+1)+C_{T s} \Phi x(k)-\beta x_{3} \frac{q_{c}}{p_{c}}(k+1) \\
+S(k)-\alpha T S(k)+f_{0}+\delta_{0} \operatorname{sgn}(S(k))
\end{array}\right]
$$

\subsection{Stability analysis on DTSMC law}

The stability condition for DTSMC law in (27) is necessary to prove the movement of the state trajectory along the sliding surface must be terminated at a stable origin. Considering Sarpturk stability analysis in [48] which gives the inequalities as (28) and (29).

$$
\begin{aligned}
& {\left[S_{T}(k+1)-S_{T}(k)\right] \operatorname{sgn}\left(S_{T}(k)\right)<0} \\
& {\left[S_{T}(k+1)+S_{T}(k)\right] \operatorname{sgn}\left(S_{T}(k)\right)>0}
\end{aligned}
$$


using (26) and (27), the lower limit of Sarpturk stability condition in (28) can be expressed as:

$$
-\alpha T\left|S_{T}(k)\right|-\varepsilon T-2 \delta_{0}<0
$$

from (26) and (27), the upper limit of Sarpturk stability condition in (29) can be expressed as:

$$
2 S_{T}(k)-q t\left|S_{T}(k)\right|-\varepsilon t>0
$$

from both conditions (30) and (31), it can be concluded that, the DTSMC law is stable when $\delta_{0}>0$.

\section{SIMULATION}

In order to demonstrate the performance of the DSMC controller, simulations are conducted based on diving plane EOM of NPS AUV II as shown in Figure 1 and dynamics model EOM in (17). Considering the nominal model parameters are given in Table 3 with parametric uncertainty and external disturbances effect on the model dynamics as in (16). All controller parameters given in Table 4.

Table 3. The NPS AUV II model parameter [40]

\begin{tabular}{ccc}
\hline Parameter & Value & Units \\
\hline$m$ & 5443.4 & $\mathrm{Kg}$ \\
$W$ & 53400 & $\mathrm{~N}$ \\
$Z_{G}$ & 0.061 & $\mathrm{M}$ \\
$I_{y}$ & 13587 & $\mathrm{Nms}^{2}$ \\
$M_{\dot{q}}$ & $-1.7 \times 10^{-2}$ & $\mathrm{Kg}$ \\
\hline
\end{tabular}

Table 4. Controller's parameters

\begin{tabular}{clll}
\hline Control algorithm & \multicolumn{3}{c}{ Table column head } \\
\hline PID & $\boldsymbol{k}_{\boldsymbol{p}}=-\mathbf{0 . 0 9}, \quad \boldsymbol{k}_{\boldsymbol{d}}=-\mathbf{0 . 0 1}, \quad \boldsymbol{k}_{\boldsymbol{i}}=\mathbf{0 . 2 3}$ \\
DSMC & $\varepsilon=0.0016, \quad T=0.2 \mathrm{~s}, \quad p=1$, & \\
& $C_{S}=\left[\begin{array}{llll}0.001822 & 0.70 & 0.27-0.0000001\end{array}\right]$ \\
DTSMC & $\varepsilon=0.0016, \quad T=0.2 \mathrm{~s}, \quad p=1, \quad \beta=3, \quad \frac{q_{c}}{p_{c}}=\frac{13}{15}$ \\
& $C_{T S}=\left[\begin{array}{llll}0.257 & 120 & 134 & -0.0012\end{array}\right]$ & \\
\hline
\end{tabular}

The performance of DTSMC, DSMC and PID controller in Figure 2 until Figure 8 are measured according to AUV position tracking and pitch angle response, chattering effect in the control input, position error convergence and rapidity of the sliding surface respectively. Commercial PID controller has faster rise time than DSMC and DTSMC for position tracking and pitch angle. However, the control output system for both position tracking and pitch angle show explicit overshoot with longer settling time as demonstrated in Figure 2 and Figure 3. Opposite to DSMC and DTSM shows no overshoot and shorter settling time. This is because, SMC family is kind of robust type controller to parameter variations and disturbances.

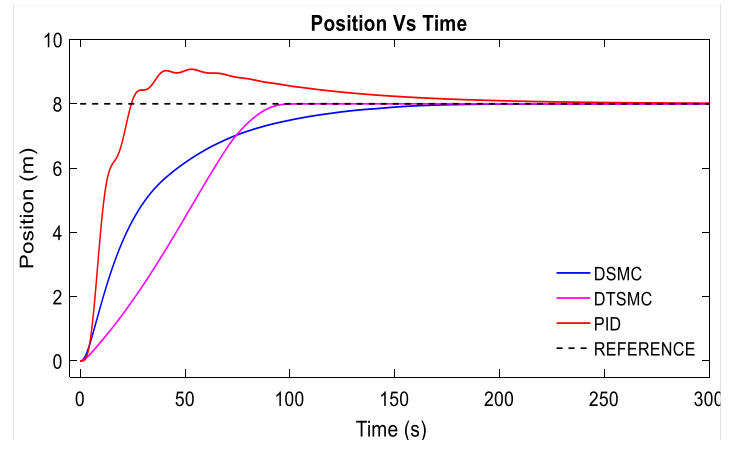

Figure 2. Position tracking of AUV for all controllers

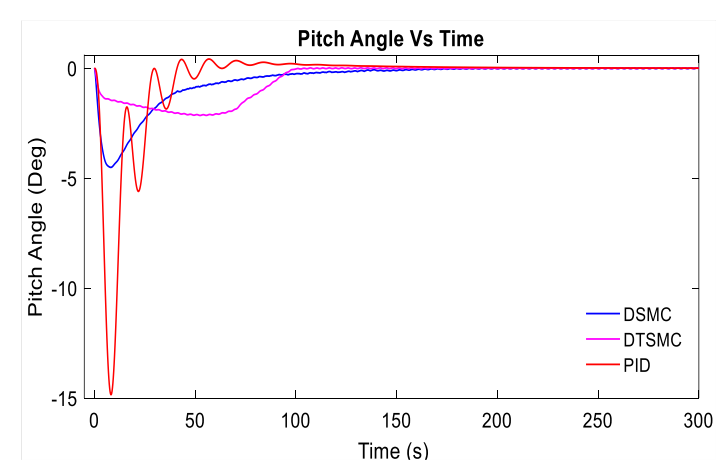

Figure 3. Pitch angle of AUV for all controllers 
The chattering effect in the control input clearly can be seen in DSMC and significantly reduced in DTSMC, while none in PID as illustrated in Figure 4 and Figure 5. This is because, in DTSMC the chattering effect supressed by nonlinear component in which the convergence speed of the system state to the equilibrium point is accelerated. However, in DSMC the system state forced to the sliding surface and asymptotically slide towards equilibrium point. The asymptotical characteristic during sliding mode phase prolong the convergence process as demonstrated in Figure 6. Therefore, the chattering effect in DSMC is higher than DTSMC. Different in PID controller where feedback mechanism is employed in the closed loop system and none of the switching function component introduced in the PID control law.

Although DTSMC has faster error convergence as in Figure 6 however, the reaching phase is slower than DSMC as shown in Figure 7 and Figure 8 respectively. Thus, the robustness of DTSMC model in (27) does not surpass the DSMC model in (24). The fractional power rule in nonlinear sliding surface tend to reduce the magnitude of the convergence rate when the system state far away from equilibrium point. In other word, the convergence performance of DTSMC is sensitive to the system state initial conditions.

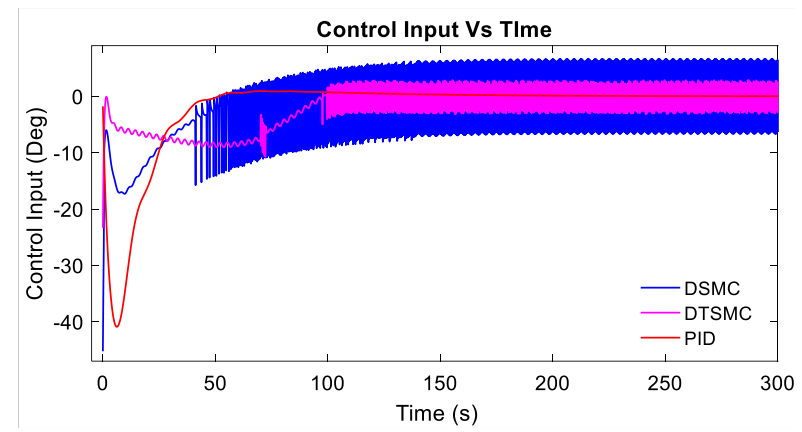

Figure 4. Control input of AUV for all controllers

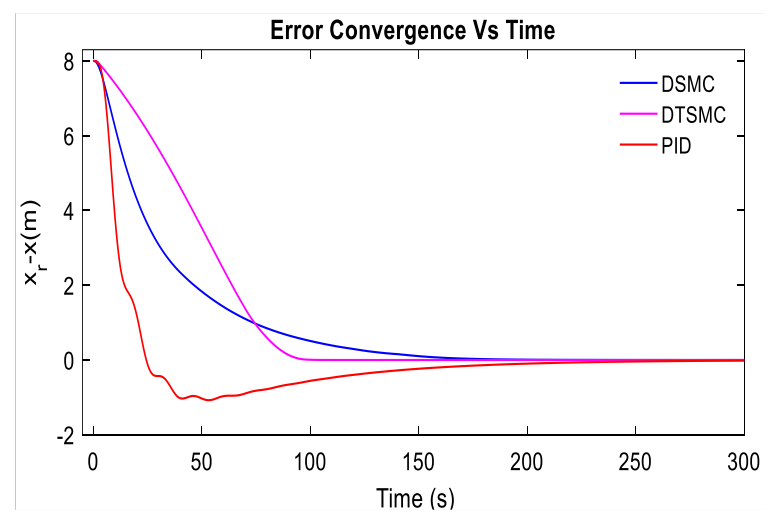

Figure 6. Convergence speed for all controllers

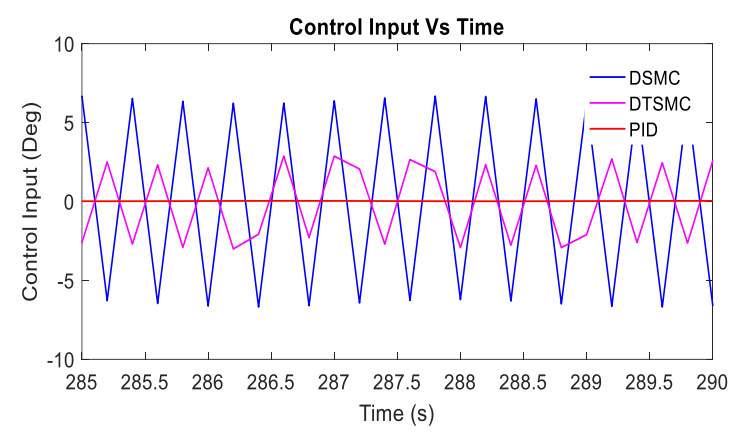

Figure 5. Chattering in DSMC and DTSMC

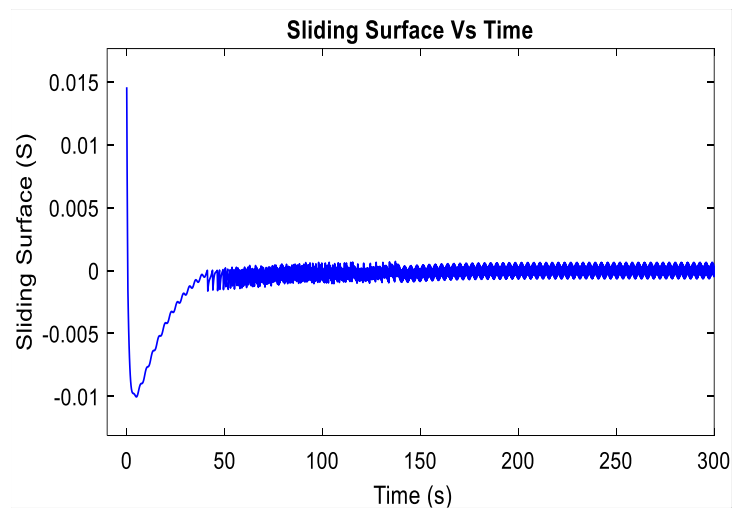

Figure 7. Sliding surface for DSMC

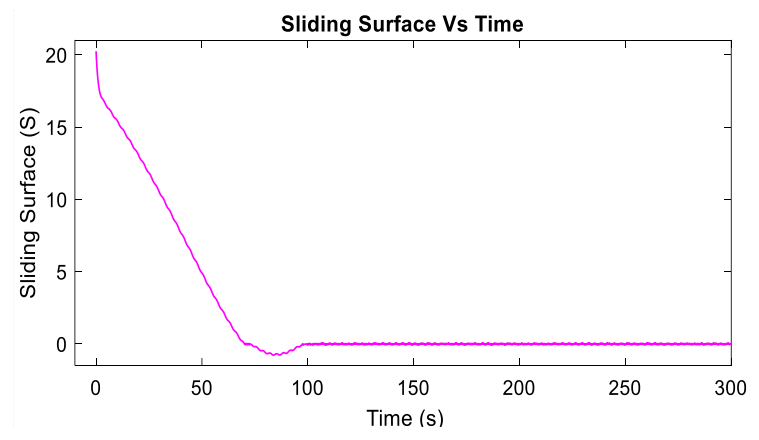

Figure 8. Sliding surface for DTSMC 


\section{CONCLUSION}

DTSMC which has nonlinear sliding surface applied to the diving motion of NPS AUV II intentionally to improve the dynamic response of the closed loop system. The fractional power in nonlinear component speed up the convergence acceleration and reduce the chattering effect at the same time. However bad convergence performance when the state far away from the equilibrium point. To overcome this problem, another component will be added to the terminal sliding surface design known as linear attractor. For this reason, Discrete Fast Terminal Sliding Mode Control (DFTSMC) came into this research which will be discuss on another chapter.

\section{ACKNOWLEDGEMENTS}

The authors would like to thank Universiti Tun Hussein Onn Malaysia (UTHM) for TIER 1 grant Vot H148, GPPS grant Vot H316 and AdMiRe FKEE for their support.

\section{REFERENCES}

[1] R. G. Damian, N. Jula, and S. V. Paţurcă, "Autonomous underwater vehicles - achievements and current trends," Scientific Bulletin" Mircea cel Batran" Naval Academy., vol. XXI, no. 1, pp. 85-89, 2018.

[2] S. Heshmati-Alamdari, A. Nikou, and D. V. Dimarogonas, "Robust trajectory tracking control for underactuated autonomous underwater vehicles," in 2019 IEEE 58th Conference on Decision and Control (CDC), 2019, pp. 83118316.

[3] R. Yang, Modeling and robust control approach for autonomous underwater vehicles, Ocean University of China, 2016.

[4] R. Yang, I. Probst, A. Mansour, M. Li and B. Clement, "Underwater vehicle modeling and control application to ciscrea robot underwater vehicle modeling and control application to ciscrea robot," Quantitative Monitoring of the Underwater Environment., pp. 89-106, 2016.

[5] V. Upadhyay, S. Gupta, A. C. Dubey, et al., "Design and motion control of autonomous underwater vehicle, amogh,” 2015 IEEE Underw. Technol. UT 2015, 2015, pp. 1-9.

[6] R. Cui, C. Yang, Y. Li, and S. Sharma, "Adaptive Neural Network Control of AUVs with Control Input Nonlinearities Using Reinforcement Learning," IEEE Transactions on Systems, Man, and Cybernetics: Systems, vol. 47, no. 6, pp. 1019-1029, 2017.

[7] X. Yang, J. Yan, C. Hua, and X. Guan, "Trajectory Tracking Control of Autonomous Underwater Vehicle With Unknown Parameters and External Disturbances," IEEE Transactions on Systems, Man, and Cybernetics: Systems, vol. 51, mo. 2, pp. 1054 - 1063, 2021.

[8] P. S. Londhe, D. D. Dhadekar, B. M. Patre, and L. M. Waghmare, "Non-singular terminal sliding mode control for robust trajectory tracking control of an autonomous underwater vehicle," 2017 Indian Control Conf. ICC 2017 Proc., no. Icc, 2017, pp. 443-449.

[9] L. Qiao and W. Zhang, "Adaptive second-order fast nonsingular terminal sliding mode tracking control for fully actuated," IEEE Journal of Oceanic Engineering, vol. 44, no. 2, pp. 363-385, 2018.

[10] J. E. Refsnes, A. J. Srensen, and K. Y. Pettersen, "Output feedback control of slender body underwater vehicles with current estimation," International Journal of Control, vol. 80, no. 7, pp. 1136-1150, 2007.

[11] A. Adhami-Mirhosseini, A. P. Aguiar, and M. J. Yazdanpanah, "Seabed tracking of an autonomous underwater vehicle with nonlinear output regulation," 2011 50th IEEE Conference on Decision and Control and European Control Conference, 2011, pp. 3928-3933.

[12] Y. Liu, P. Fang, D. Bian, H. Zhang, and S. Wang, "Fuzzy comprehensive evaluation for the motion performance of autonomous underwater vehicles," Ocean Engineering, vol. 88, pp. 567-577, 2014.

[13] P. Chen, Y. Li, Y. Su, X. Chen, and J. Yanqing, "Review of AUV Underwater Terrain Matching Navigation," The Journal of Navigation., vol. 68, no. 6, pp. 155-1172., 2015.

[14] H. F. Ghavidel and A. A. Kalat, "Robust control for MIMO hybrid dynamical system of underwater vehicles by composite adaptive fuzzy estimation of uncertainties," Nonlinear Dynamics, vol. 89, no. 4, pp. 2347-2365, 2017.

[15] B. Sun, D. Zhu, F. Ding, and S. X. Yang, "A novel tracking control approach for unmanned underwater vehicles based on bio-inspired neurodynamics," J. Mar. Sci. Technol., vol. 18, no. 1, pp. 63-74, 2013.

[16] X. P. and Z. P. Shiqiang Zhu, Xinglai Jin, Bin Yao, Qingcheng Chen, "Non-linear sliding mode control of the lower extremity exoskeleton based on human-robot cooperation," International Journal of Advanced Robotic Systems, vol. 13, no. 5, pp. 1-10, 2016.

[17] M. S. M. Aras, S. S. Abdullah, H. I. Jaafar, M. A. A. Aziz, and A. M. Kassim, "Tuning process of single input fuzzy logic controller based on linear control surface approximation method for depth control of underwater remotely operated vehicle," Journal of Engineering and Applied Sciences, vol. 8, no. 6, pp. 208-214, 2013.

[18] W. Y. Gan, D. Q. Zhu, W. L. Xu, and B. Sun, "Survey of trajectory tracking control of autonomous underwater vehicles," Journal of marine science and technology, vol. 25, no. 6, pp. 722-731, 2017.

[19] Y. Shi, C. Shen, H. Fang, and H. Li, "Advanced control in marine mechatronic systems : A survey," IEEE/ASME Transactions on Mechatronics, vol. 22, no. 3, pp. 1121-1131, 2017.

[20] Q. Xu, H. P. Du, B. He, T. H. Yan, W. H. Li, and S. S. Sun, "A novel reaching law for sliding mode control of uncertain discrete-time systems," Mathematical Problems in Engineering, 2018. 
[21] H. Ma, J. Wu, and Z. Xiong, "A novel exponential reaching law of discrete-time sliding-mode control," IEEE Transactions on Industrial Electronics, vol. 64, no. 5, pp. 3840-3850, 2017.

[22] P. Padmalatha and S. Thomas, "Discrete time reaching law based variable structure control for fast reaching with reduced chattering," IAES International Journal of Robotics and Automation, vol. 9, no. 1, pp. 51-61, 2020.

[23] M. S. G. Dias, Discrete time sliding mode control strategies applied to a multiphase brushless DC machine, Kassel University, 2017.

[24] L. Mo, Y. Liu, and Y. Zhang, "Sliding mode variable structure control for surface permanent magnet synchronous motors based on a fuzzy exponential reaching law," Mathematical Problems in Engineering, 2019.

[25] Z. Wang, S. Li, and Q. Li, "Discrete-time fast terminal sliding mode control design for DC-DC buck converters with mismatched disturbances," IEEE Transactions on Industrial Informatics, vol. 16, no. 2, pp. 1204-1213, 2019.

[26] S. Amirkhani, S. Mobayen, N. Iliaee, O. Boubaker, and S. H. Hosseinnia, "Fast terminal sliding mode tracking control of nonlinear uncertain mass-spring system with experimental verifications," International Journal of Advanced Robotic Systems, vol. 16, no. 1, pp. 1-10, 2019.

[27] K. A. J. Xu, Advanced discrete- time control. Singapore: Springer, 2015.

[28] Q. Xu and Z. Cao, "Piezoelectric positioning control with output- based discrete-time terminal sliding mode control," IET Control Theory \& Applications, vol. 11, no. 5, pp. 694-702, 2017.

[29] W. Shikai, J. Hongzhang, and M. Lingwei, "Trajectory tracking for underactuated UUV using terminal sliding mode control," In 2016 Chinese Control and Decision Conference (CCDC), 2016, pp. 6833-6837.

[30] Z. Yangyang, G. Li'E, L. Weidong, and L. Le, "Research on control method of AUV terminal sliding mode variable structure," 2017 International Conference on Robotics and Automation Sciences (ICRAS), 2017, pp. 8893.

[31] T. Elmokadem, M. Zribi, and K. Youcef-toumi, "Terminal sliding mode control for the trajectory tracking of underactuated Autonomous Underwater Vehicles," Ocean Engineering, vol. 129, pp. 613-625, 2017.

[32] L. Qiao and W. Zhang, "Adaptive second-order fast nonsingular terminal sliding mode tracking control for fully actuated autonomous underwater vehicles," IEEE Journal of Oceanic Engineering, vol. 44, no. 2, pp. 363-385, 2018.

[33] S. Majidabad, "Discrete-time terminal sliding mode control of chaotic lorenz," Journal of Control and Systems Engineering, vol. 1, no. 1, pp. 1-8, 2013.

[34] B. Wang, "On discretization of sliding mode control systems on discretization of sliding mode control systems," Phd Thesis, RMIT University Melbourne, Australia, 2008.

[35] S. Janardhanan and B. Bandyopadhyay, "On discretization of continuous-time terminal sliding mode," IEEE transactions on automatic control, vol. 51, no. 9, pp. 1532-1536, 2006.

[36] X. Yu, J. Xu, Y. Hong, and S. Yu, "Analysis of a class of discrete-time systems with power rule," Automatica, vol. 43 , no. 3, pp. $562-566,2007$.

[37] K. Abidi, J. X. Xu, and J. H. She, "A discrete-time terminal sliding-mode control approach applied to a motion control problem," IEEE Transactions on Industrial Electronics, vol. 56, no. 9, pp. 3619-3627, 2009.

[38] Z. Galias and X. Yu, "Dynamical behaviors of discretized second-order terminal sliding-mode control systems," IEEE Transactions on Circuits and Systems II: Express Briefs, vol. 59, no. 9, pp. 597-601, 2012.

[39] S. Li, H. Du, and X. Yu, "Discrete-time terminal sliding mode control systems based on euler's discretization," IEEE Transactions on Automatic Control, vol. 59, no. 2, pp. 546-552, 2014.

[40] T. I. Fossen, "Guidance and control of ocean vehicles," 4th ed., New York: John Willey \& Sons, 1994

[41] A. J. Healey and D. B. Marco, "Slow speed flight control of autonomous underwater vehicles. Experimental results with NPS AUV II," in The Second International Offshore and Polar Engineering Conference, 1992, pp. 523-532.

[42] A. J. Healey and D. Lienard, "Multivariable sliding-mode control for autonomous diving and steering of unmanned underwater vehicles," IEEE journal of Oceanic Engineering, vol. 18, no. 3, pp. 327-339 1993.

[43] E Snam, "Nomenclature for treating the motion of a submerged body through a fluid," The Society of Naval Architects and Marine Engineers, Technical and Research Bulletin, pp. 1-5, 1950.

[44] B. Jalving, "The NDRE-AUV Flight Control System," IEEE Journal of Oceanic Engineering, vol. 19, no. 4. pp. 497-501, 1994.

[45] T. I. Fossen, "Marine control systems: guidance, navigation and control of ships, rigs and underwater vehicles," Norway: Marine Cybernetics, 2002.

[46] P. S. Londhe, D. D. Dhadekar, B. M. Patre, and L. M. Waghmare, "Uncertainty and disturbance estimator based sliding mode control of an autonomous underwater vehicle," International Journal of Dynamics and Control, vol. 5, no. 4, pp. 1122-1138, 2017.

[47] W. Gao, Y. Wang, and A. Homaifa, "Discrete-time variable structure control systems," IEEE transactions on Industrial Electronics, vol. 42, no. 2, pp. 117-122, 1995.

[48] S. Sarpturk, Y. Istefanopulos, and O. Kaynak, "On the stability of discrete-time sliding mode control system," IEEE Transactions on Automatic Control., vol. 32, no. 10, pp. 930-932, 1987. 


\section{BIOGRAPHIES OF AUTHORS}
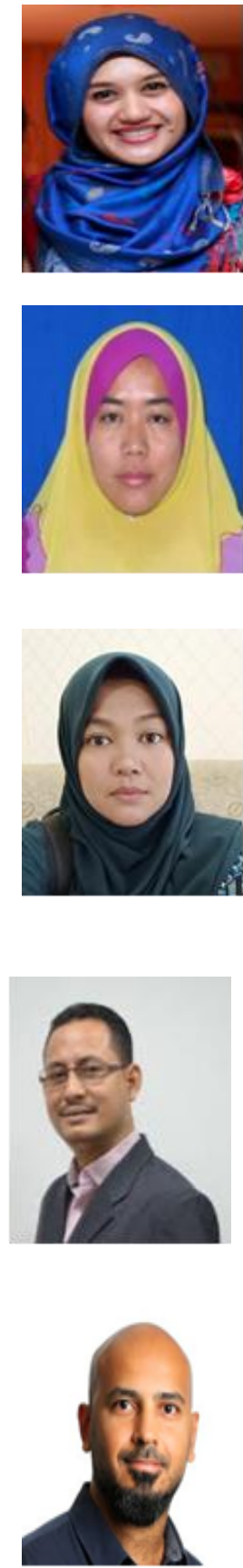

Nira Mawangi Sarif received her Bachelor of Engineering from Strathclyde University United Kingdom in 2005 in Electrical (Power) Engineering. From 2005 until 2007, she worked as Material Analysit Engineer at Flextronics Sdn Bhd. She than work at Bumi Arada Berhad (BAB) as Lead Procuement Engineer from 2007 until 2017 specialized in Supply Chian Management and frame agreement. Currently, she is pursuing her Master in Electrical and Electronic Engineering at University Tun Hussein Onn (UTHM). Her research interests include robust control of underwater vehicle.

Rafidah Ngadengon received her Bachelor of Information Engineering from Kitami Institute of Technology, Japan. She obtained Masters in Electrical Engineering (Mechatronics) and $\mathrm{PhD}$ degree in Electrical Engineering from Universiti Teknologi Malaysia (UTM), Malaysia in 2005 and 2017 respectively. Her research interests are in robust control, system identification and intelligent control.

Herdawatie Abdul Kadir graduated from the Universiti Teknologi Malaysia, in 2001 with a Bachelor in Electrical (Electronic) Engineering. From 2001 until 2003, she worked as an Instructor in Universiti Tun Hussein Onn Malaysia (UTHM). She then pursued her MEng. Mechatronic and Automatic Control at the Universiti Teknologi Malaysia, graduating in 2004. Since then, she has been working at the Universiti Tun Hussein Onn Malaysia (UTHM), as a full-time lecturer. Currently, she pursuing her PhD in Electrical and Electronic Engineering at Universiti Sains Malaysia (USM), in a field of Computational Intelligence. Her current research interests are automatic control, localization, mapping and cooperative system

Mohd Hafiz bin A. Jalil received his Barchelor of Engineering in Electrical Engineering from Universiti Teknologi MARA (UiTM) in 2003. He received his MSc. in Electrical Engineering (Mechatronic) from Universiti Teknologi Malaysia (UTM) in 2006 and his $\mathrm{PhD}$ in Electrical Engineering from Universiti Teknologi MARA in 2019. He has a lecturer at Faculty of Electrical and Electronic Engineering, Universiti Tun Hussein Onn Malaysia (UTHM) since 2006. His research interest is related with process control, application of advanced control, instrumentation and system identification.

Dr Khalid Abidi received his Bachelor degree in Mechanical Engineering from the Middle East Technical University, Ankara, Turkey in 2002 and the Masters degree in Electrical Engineering and Computer Science from Sabanci University, Istanbul, Turkey in 2004. He obtained his PhD degree in Electrical and Computer Engineering, specializing in the area of Control Engineering, from the National University of Singapore in 2009. Dr Abidi is currently a lecturer of Electrical Power Engineering at Newcastle University based in Singapore. Prior to joining Newcastle University Dr Abidi worked as an Assistant Professor of Mechatronics Engineering at Bahcesehir University, Istanbul, Turkey from September 2009 until June 2014. 